NASA Contractor Report 185178

\title{
Thermocapillary Migration of Liquid Droplets in a Temperature Gradient in a Density Matched System
}

N. Rashidnia

Sverdrup Technology, Inc.

NASA Lewis Research Center Group

Cleveland, Ohio

and

R. Balasubramaniam

Case Western Reserve University

Cleveland, Ohio

December 1989

Prepared for

Lewis Research Center

Under Contract NAS3-25266 and Cooperative Agreement NCC3-140

\section{N/Sก}

National Aeronautics and

Space Administration

(NASA-CR-135170) THERMOCAPILLARY MIGPATION

N90-14512

UF LIOUIU ORUPLETS IN A TFMPERATURE GRAOIFNT

IN A DENSITY MATCHED SYSTEM I INAI RepOrt

(svertrup Tectonoloyy) $28 \mathrm{p}$

CSCL 200

$\begin{array}{rl}\text { Unclas } \\ 63 / 34 & 0252592\end{array}$ 
$+$. 


\section{THERMOCAPILLARY MIGRATION OF LIQUID DROPLETS IN A TEMPERATURE GRADIENT IN A DENSITY MATCHED SYSTEM \\ N. Rashidnia \\ Sverdrup Technology, Inc. \\ NASA Lewis Research Center Group \\ Clevel and, Ohio 44135 \\ and

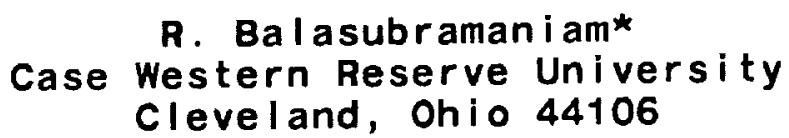

ABSTRACT

An experimental investigation of thermocapillary flow in droplets of a vegetable oil (partially

hydrogenated soybean oil) immersed in silicone oil was conducted in a test cell with a heated top wall and a cooled bottom wall. The liquids are nearly immiscible and have equal densities at a temperature below the room temperature, thus providing a simulation of low-gravity conditions by reducing the buoyancy forces.

The interfacial tension between the two oils was measured in the temperature range $20-50^{\circ} \mathrm{C}$ using a capil-

lary tube and $\frac{d \sigma}{d T}$ was determined to be negative. Droplets ranging in sizes from $3 \mathrm{~mm}$ to $1 \mathrm{~cm}$ diameter were

injected into the silicone oil. The vertical temperature profile in the bulk liquid (silicone oil) produces temperature variations along the interface which induce variations in the interfacial tension. The flow inside the droplet driven by the resulting interfacial shear stresses was observed using a laser light- sheet flow visualization technique. The flow direction is consistent with the sign of $\frac{d \sigma}{d T}$. The observed maximum surface velocitics are compared to the theoretical predictions of Young et al. (1959).

*NASA Resident Research Associate at Lewis Research Center. 
For short times after injection, the droplets were driven by this flow towards the hot wall above the matched-density temperatures until the droplet reached a point where the forces due to the flow and buoyancy were equal. After longer times, the droplets moved to the cold side due to suspected density changes caused by mass transfer from the droplets to the silicone oil. This was confirmed by tests under isothermal conditions, where it was observed that droplets of all sizes fell to the cold bottom eventually.

Thus, even though the thermocapillary flow inside the droplets persisted for long times in spite of the mass transfer, the migration of droplets towards the hot side was eventually affected by uncontrolled buoyancy forces resulting from density changes due to mass transfer. While additional liquids are being tried, it is suggested from the present experience that reduced gravity experiments will probably be necessary to provide unambiguous data for the migration of droplets.

\section{Introduction}

The investigation of the migration of droplets in liquids due to thermocapillary induced flow is significant not only in enhancing the understanding of such flows but also has direct application to material sciences and chemical engineering. It has been suggested that the low gravity environment available in space orbit can be exploited to provide advancements in materials processing (Neilson and Weinberg, 1977, Weinberg, 1978). For example, containerless manufacturing of high technology ultrapure materials may be possible in such an environment because of the elimination of heterogeneous reaction and nucleation effects at 
the container walls, allowing larger undercooling of the melts, and substantially reducing the oftentimes deleterious effects of time-dependent buoyancy induced flows. However, such space manufacturing endeavors involve complex, non-equilibrium systems oftentimes with multiple interfacial regions providing the potential for significant surface tension driven flows which could also be detrimental to product quality. Thus, it is imperative to develop an understanding of such effects through a coordinated earth-space research effort. The ground-based experimental study reported here was conducted to support such an effort in one special area - thermocapillary migration of immiscible droplets/bubbles in a continuous phase liquid.

The migration of bubbles, or droplets, is an important aspect of many engineering sciences and space manufacturing issues. It is a prime candidate for many studies as it is without complications such as frec, or moving contact lines. In material science processes as in glass manufacturing, etc., gas bubbles can be formed from the byproducts of chemical reactions or gas trapped in the interstices of the raw material. In a 1-g environment the migration of the bubbles due to buoyancy effects can be utilized as a separation mechanism. However, in a microgravity environment of space other forces must be utilized for the separation. Young et al. (1959) pointed out that a fluid droplet placed in a thermal gradient environment will experience a force due to the induced capillary flow. The interfacial tension will typically be minimum at the warm pole of the droplet, and will increase toward the cooler pole. The resulting tangential stress will drag the neighboring continuous phase fluid toward the cooler pole, and the reaction on the droplet will therefore be in the opposite direction. If the droplet is free to move, it will migrate toward warmer regions as a conse- 
quence, and hence provide a separation mechanism sensitive to the magnitude and direction of the bulk temperature gradient. After the pioneering work of Young et al. (1959), the Marangoni convection of bubbles in liquids was subject to many experimental investigations (Papazian and Wilcox, 1978, Hardy, 1979, Thompson, 1979, Nähle et al., 1987). The investigations of the thermocapillary migration of droplets in liquid are more recent and less have been performed. Chifu et al. (1983) observed the flow on the surface of a droplet (freely suspended in a liquid) by injecting a small amount of a dyed surfactant into the interface. The velocity of the spreading surfactant front was measured and compared with the values resulting from their theoretical model. By also, using a two component system with a miscibility gap (methanol/cyclohexane) and cooling it down below the temperature where cyclohexane separates, they observed a thermocapillary migration of the separated droplets in the direction of the warmer region. The thermocapillary migration of water droplets in butyl benzoate was investigated by Delitzsch et al. (1984). The two components of the liquid system that they used were immiscible and have the same density at about $32^{\circ} \mathrm{C}$. Water droplets injected into a stably stratified layer of butyl benzoate sorted out according to their diameters in a manner that smaller droplets migrated to higher temperatures than the larger ones. The final droplet positions was determined by the balance of the thermocapillary (proportional to the surface area) and buoyancy forces (proportional to the volume). Seikmann et al. (1986) also used water droplets in silicone oil in a microgravity environment (D1 shuttle mission), but did not observe thermocapillary migration. Barton and Subramanian (1989), observed thermocapillary convection of ethyl salicylate droplets in diethylene glycol for Marangoni and Reynolds numbers much less than one. 
In order to continue to increase the understanding of these phenomena it was decided that an important step would be to elucidate the flow inside and on the interface of a droplet, and its migration as well as its equilibrium position in a vertical temperature gradient. The visualization of the flow pattern would be an added confirmation that the mechanism for the migration is a result of the surface induced thermocapillary flow (in the opposite direction to the migration) and not due to any bulk induced motion (like buoyancy induced flow, for example, which would be in the same direction). Since conflicting results have been obtained using water systems, it was decided to use an easily available matched-density (at $15^{\circ} \mathrm{C}$ ) liquid-liquid system consisting of vegetable oil as the droplet liquid and 5 CS. silicone oil as the continuous phase liquid.

\section{Theoretical Considerations}

To our knowledge, there is no theoretical treatment of thermocapillary migration of droplets for nonnegligible Marangoni numbers. One choice for the purpose of comparison of experimental results is to refer to the classical linear theory of Young et al. (1959) as a guideline. They obtained the migration velocity V of the droplets as

$$
V=\frac{D^{2} g\left(\rho-\rho^{\prime}\right)\left(\mu+\mu^{\prime}\right)}{6 \mu\left(2 \mu+\mu^{\prime}\right)}-\frac{d T}{d z} \quad \frac{d \sigma}{d T} \frac{D \alpha}{\left(2 \mu+3 \mu^{\prime}\right)\left(2 \alpha+\alpha^{\prime}\right)}
$$


where $D$ is the droplet diameter, $g$ the gravitational acceleration, $\frac{d T}{d z}$ the temperature gradient at infinity, $\frac{d \sigma}{d T}$ the thermal interfacial tension coefficient, $\mu$ and $\mu^{\prime}$ the dynamic viscosities and $\alpha$ and $\alpha^{\prime}$ the thermal diffusivities of the continuous phase fluid and the droplet liquid respectively. In the above equation and in what follows, a prime (') designates the property in the droplet phase. The migration velocity is the sum of two terms, the first representing the velocity of a droplet due to buoyancy forces and the second term describing the velocity induced by Marangoni forces. When the droplet reaches a stationary position, Marangoni and gravity forces balance each other and $\mathrm{V}=0$.

$$
\text { Substituting } \rho=\rho_{\mathrm{m}}\left[1-\beta\left(T_{\mathrm{eq}}-T_{\mathrm{m}}\right)\right], \rho^{\prime}=\rho_{\mathrm{m}}\left[1-\beta^{\prime}\left(T_{\mathrm{eq}}-T_{\mathrm{m}}\right)\right], \alpha=\frac{k}{\rho C_{\mathrm{p}}} \text {, and } \alpha^{\prime}=\frac{k^{\prime}}{\rho^{\prime} C_{\mathrm{p}}{ }^{\prime}} \text {. }
$$

(where $k, C_{\mathrm{p}}, k^{\prime}, C_{\mathrm{p}}^{\prime}$ are the heat conduction coefficient and specific heat for continuous phase and the droplet liquids respectively) into the above equation, one can write:

$$
T_{\mathrm{eq}}-T_{\mathrm{m}}=K\left(\frac{1}{D}\right) \frac{d T}{d z}
$$

$$
\text { where } K=\frac{6 \mu\left(-\frac{d \sigma}{d T}\right)}{\rho_{\mathrm{m}} g\left(\mu-\mu^{\prime}\right)\left(2+\frac{k^{\prime}}{k}\right)\left(\beta-\beta^{\prime}\right)} \text { is a material constant. Therefore the temperature dif- }
$$

ference from the matched density temperature $\left(T_{\mathrm{eq}}-T_{\mathrm{m}}\right)$ at which the droplet is stationary $\left(T_{\mathrm{eq}}\right)$ is a 
function of two controllable variables, namely $\frac{1}{D}$, and $\frac{d T}{d z}$. If $\frac{d T}{d z}$ is held constant, all droplets of equal size should experience the same deflection from the matched density temperature level. On other hand, if $\frac{d T}{d z}$

were to be increased, the above deflection would be larger. In the present study, experiments were performed to measure $\left(T_{\mathrm{eq}}-T_{\mathrm{m}}\right)$ with $D$ and $\frac{d T}{d z}$ as controlled variables.

The maximum velocity on the interface according to Youngs theory occurs at the equator of the droplet, and is expressed as follows:

$$
V \max =\frac{\left(-\frac{d \sigma}{d T}\right) \frac{d T}{d z}\left(\frac{D}{2}\right)}{\left(\mu+\mu^{\prime}\right)\left(2+\frac{k^{\prime}}{k}\right)}
$$

This has been used for a comparison to our measured interfacial velocities on the droplet equator.

\section{Experimental Apparatus, Test Liquids and Procedure}

\subsection{Experimental Apparatus}

The apparatus used in these experiments is shown in Figure 1. It consists of a rectangular cell filled with the tcst fluid with a movable top wall. The top and bottom walls are made from two copper plates, and 
the side walls are made from transparent $1 / 2^{n}$ thick plexiglass. Plexiglass has low conductivity and permits good flow observation. Copper tubes, of $3 / 8^{\prime \prime}$ diameter, shaped in a square coil are soldered outside the cell to both the bottom and the adjustable upper plates (milled and polished surfaces of $1 / 4 " \times 5 " x 5 ", 1 / 4 " x 4^{\prime \prime} \times 4^{\prime \prime}$ copper plates) for cooling and heating purposes. The tubes of each plate are connected to separate constant temperature baths so as to adjust and maintain the temperatures of the plates. The four vertical walls are fixed to the bottom wall and sealed to prevent any possible leakage. The upper wall is attached from the top side on an adjustable column to enable changes in the distance between the copper plates. The upper wall also has two $1 / 4^{\prime \prime}$ diameter holes, one in the center and another $1^{\prime \prime}$ away from the center and side wall. These boles are used to fill the test cell with the continuous phase fluid, and also to provide access to position thermocouples for temperature measurements. The lower copper plate has a 1/8" diameter hole at the center with a drain valve attached to it from the outside. A compartment is, therefore, created between the copper plates. It is within this chamber that the experiments occur. In order to achieve a stably stratified liquid layer without any unwanted flows due to buoyancy, the upper plate was maintained at a higher temperature than the lower plate in all of the experiments.

An injector was designed to produce droplets from 0.5 to $10 \mathrm{~mm}$ diameter. The injector is a $0.25 \mathrm{~mm}$ diameter and $3^{\prime \prime}$ long hypodermic needle and was connected, via a $1 / 8^{\prime \prime}$ diameter tygon tubing, to a syringe, which in turn, was supported by a holder at about the injection level. The syringe was manually actuated to produce the droplets. The injector hole in the side wall is placed at about the location of the matched-den- 
sity temperature of the liquid-liquid system used in this experiment, and is sealed with a rubber cork. After a single droplet of a desirable size is generated the injector was very carefully pulled out of the side wall of the test cell to deploy the droplet. A laser light-sheet flow visualization technique has been used to study the flow field in the droplets. The optical components for this technique consist of a $0.5 \mathrm{~mm}$ laser light beam passing through a cylindrical lens, thereby generating a sheet of light $0.3 \mathrm{~mm}$ in width. The light-sheet can be used to illuminate flow tracer particles introduced in the flow field of interest. The motion of the particles (1 micron aluminum oxide) reflecting the laser light can be observed and recorded on film and video cassette tapes for further analysis. This technique was used to observe the flow patterns and to estimate the droplet interface maximum velocities.

\subsection{Test Liquids}

Quite by accident we found that a nearly immiscible, non-toxic liquid which had a density very close to the 5 CS. silicone oil is vegetable oil. Therefore we chose this as the droplet phase. We measured most of the necessary liquid properties in our laboratory. The matched density temperature for the experiments reported here was measured in two ways: 1) Using a Mohr-Westphal specific gravity meter along with a HAAKE F4-C temperature bath, densities of both liquids vs. temperature were measured separately, and the best fit lines over the measured temperature range were obtained. The intersection of the two fitted lines was chosen as the matched-density temperature. This was at $T_{\mathrm{m}}=15^{\circ} \mathrm{C}$ where $\rho_{\mathrm{m}}=0.911 \frac{\mathrm{g}}{\mathrm{cc}}$ (also 
$\beta=0.001197 \frac{\mathrm{cm}}{\mathrm{cm}} \frac{1}{{ }^{\circ} \mathrm{C}}$ and $\beta^{\prime}=0.000703 \frac{\mathrm{cm}}{\mathrm{cm}} \frac{1}{{ }^{\circ} \mathrm{C}}$ were obtained from these measurements). 2) An isothermal condition was created in two double wall containers, connected in series, by the same temperature bath. With the continuous phase liquid in the first container and the droplet liquid in the second, the bath was run until a predetermined isothermal condition in the entire volume of the liquids was achieved. The droplets were then placed into the continuous phase liquid. At temperatures not close to $T_{\mathrm{m}}\left(\left|T-T_{\mathrm{m}}\right|>\right.$ or $\left.=2^{\circ} \mathrm{C}\right)$, the droplets either fell down to the bottom $\left(T>T_{\mathrm{m}}+2^{\circ} \mathrm{C}\right)$ or floated on top $\left(T<T_{\mathrm{m}}-2^{\circ} \mathrm{C}\right)$. For temperatures close to $T_{\mathrm{m}}\left(\left|T-T_{\mathrm{m}}\right|<1^{\circ} \mathrm{C}\right.$, with $\left.T_{\mathrm{m}}=15^{\circ} \mathrm{C}\right)$, the droplets were neutrally buoyant, thus confirming the measurement of $T_{m}$ by the previous method. However, continuous mass transfer from the droplets to the silicone oil which occurred shortly after the droplets were inserted masked the precise determination of $T_{\mathrm{m}}$. In fact, in the vicinity of $T_{\mathrm{m}}$, peculiar results were observed wherein smaller droplets fell down to the bottom faster than bigger droplets, in contradistinction to the settling velocity of Hadamard and Rybczynski. Most likely, these results can be attributed to the mass transfer process. Mass transfer from the droplet oil to the silicone oil was also confirmed by observing precipitation in refrigerated samples of the silicone oil that came in contact with the vegetable oil.

It was also noticed that the matched density temperature $\left(T_{\mathrm{m}}\right)$ was changed by the aging of the vegetable oil over a few months, but the final experiments from which data is reported were conducted within 
a five day period which, to our knowledge, did not seem to change the properties of the droplet liquid.

$T_{\mathrm{m}}=15^{\circ} \mathrm{C}$ was obtained at the beginning of this five day period.

The viscosity of these liquids were measured in our laboratory at the room temperature by a Cannon-

Fenske Routine viscometer. The interfacial surface tension was measured over a temperature range of $20^{\circ} \mathrm{C}$ to $50^{\circ} \mathrm{C}$, by the use of a capillary tube (Rashidnia and Balasubramaniam, in progress). The data were suitably fitted, and the thermal interfacial tension coefficient in the best fit line over the measured temperature range was calculated to be $-0.0088 \frac{\text { dynes }}{\mathrm{cm}^{\circ} \mathrm{C}}$. An average thermal conductivity for the vegetable oil $\left(k^{\prime}=0.0003266 \frac{\mathrm{Cal}}{\mathrm{cm} \mathrm{sec}{ }^{\circ} \mathrm{C}}\right)$ was taken from the Chemical Engineering handbook (Perry and Chilton, 1973). Thermal conductivity of the silicone oil $\left(k=0.00026 \frac{\mathrm{Cal}}{\mathrm{cm} \mathrm{sec}{ }^{\circ} \mathrm{C}}\right)$ is given by DOW CORNING literature. The Prandtl number and the thermal diffusion coefficient for the continuous phase fluid are 71.75 and $0.0007 \frac{\mathrm{cm}^{2}}{\mathrm{sec}}$. Table 1 summarizes the major properties of the fluid system used for this study.

3.3 Procedure

Before each experiment, the old liquid inside the cell was drained, and the cell was wiped, cleaned, and rinsed with fresh silicone oil. The cell $\left(10 \times 10 \times 3 \mathrm{~cm}^{3}\right.$ test chamber $)$ was then assembled and filled with $5 \mathrm{CS}$. silicone oil. Then, the circulating baths were turned on to bring the upper and lower boundaries of the cell 
to the desired temperatures. This causes a vertical temperature gradient to be established in the cell. After waiting a sufficient amount of time (at least four hours) for steady state conditions to be achieved, the droplets were injected into the cell and their size, motion, and equilibrium positions observed through a Panasonic high resolution video camera and recorded on a super VHS video cassette. The diameter and position of the center of the droplets were also monitored by a cathetometer, and were manually recorded.

The temperature in the host fluid was measured by a K-type thermocouple fixed to a $0.25 \mathrm{~mm}$ moveable stem attached to a micrometer traversing mechanism. The thermocouple signals are read using a Keithly 871 Digital thermometer. Data collected from experiments using three temperature gradients are reported in this paper. For each experiment, the period from starting the temperature baths to the end of data collection lasted for about 9 continuous hours. The temperature gradient was checked at the end of each day's experiment and no measurable change (thermometer resolution $= \pm 0.1^{\circ} \mathrm{C}$ ) was detected. The temperature gradient was very linear in and beyond the range where the droplets migrated, except very close to the upper wall (within 1mm) where a small deviation occurred which could not affect the region of interest. The thermocouple was also traversed in two locations --in the center of the cell and half way to the side wall $(2.5 \mathrm{~cm}$ apart). The temperature profilesin the cell, for the two locations, were very similar confirming a one-dimensional temperature field in the cell. 
Figure 1 shows a schematic of the experiment and the optical arrangement utilized in the laser lightsheet flow visualization part of the experiment. Figure 2 is a photograph of the apparatus. The laser lightsheet flow visualization part of the experiment was conducted after the data collection was completed.

Using the same technique, we were able to obtain average maximum velocities of the particles at the interface of the droplets. The surface velocity has a maximum value at the equator (Young et al., 1959).

In all the experiments described here, the droplet temperature at equilibrium is assumed to be the same as the temperature measured on an imaginary horizontal line passing through the droplet center (using the horizontal part of the cross hair of the cathetometer) that is one inch from the vertical axis of the cell (where the droplets were normally located).

In order to obtain a measure of the magnitude of free convection flows in the cell, separate experiments were conducted. One micron size aluminum oxide particles, as tracers, were introduced into the continuous phase liquid. Sufficient time was allowed for the cell to return back to the expected steady state temperature gradient. Using a $10 \mathrm{~mW}$ power $\mathrm{He}$-Ne laser light-sheet enabled visualization of particle motion in different vertical planes of the cell. Analysis of the video recorded images and long time exposure photography showed that free convection velocitics were less than $10 \frac{\mu m}{\mathrm{sec}}$ in the worst location (up to $1 \mathrm{~mm}$ below the upper copper plate) which was along the surface direction (showing a thin cell far away from the center of 
cell where the droplets were injected and reached their equilibrium positions). This minor velocity of tracer particles could be due to a small tilt angle of the upper wall and did not seem to affect the lateral position of the droplets that were observed for more than an hour after their injection. The rest of the test cell did not show any velocities of the tracers more than $5 \frac{\mu m}{s e c}$ which were mainly downward and that was attributed to gravity induced settling.

When droplets were deployed, they were, for the most part of the experiment, located 3 to 10 radii from the horizontal walls, and 10 to 33 radii from the vertical walls of the test cell. The influence of the walls on the flow and the droplet equilibrium positions are discussed later.

\section{Results and Discussion}

All droplets analyzed were spherical, and the measured diamcters and the equilibrium temperatures were constant during the time period of the experiment wherein the measurements have been made. A typical temperature versus normalized time, $\tau=\frac{D^{2}}{\alpha^{\prime}}$, after droplet deployment is shown schematically in Figure 3. $\tau$ is the characteristic time for the droplets to reach their thermal equilibrium. After about 1.25 to $1.5 \tau$, droplets reached their maximum positions from the matched-density temperature $T_{\mathrm{m}}$. Observing this location for a short time (about $2-3 r$ ) and making sure that it did not change, this location was taken as the droplet equilibrium position, and the necessary data were recorded. This process is shown schematically in 
Figure 3. A slight plume was often observed behind the droplets resulting from dissolution of droplet liquid and the consequent changes in refractive index. Because of dissolution of some unknown component(s) that could change the density of the droplet liquid, the droplets and their central temperatures were tracked very carefully, and recorded up to the peak position shown in Figure 3. After the peak (longer times than $4 \tau$ ), droplets started to fall toward the cold wall. Typically, it took about $10 \tau$ for the droplets to fall back to the $T_{\mathrm{m}}$ level.

Figure 4 displays the measured equilibrium temperature difference from $T_{\mathrm{m}}$ versus the inverse of the droplet diameter for the three temperature gradients considered. Figure 5 shows the same data plotted as $\left(T_{\text {eq }}-T_{\mathrm{m}}\right)$ versus $\frac{\left(\frac{d T}{d z}\right)}{D}$ along with the predictions from linear theory (i.e. equation (2)). As can be seen from Figure 5, the measured data qualitatively follows the trend predicted by the linear theory, but is quantitatively quite different. The droplets seem to have migrated farther away from $\mathrm{Tm}$ when compared to the predictions. Also, the data in Figure 5 does not collapse on a single straight line for all temperature gradients and droplet sizes, as anticipated by the linear theory. Table 2 shows the measured maximum interface velocities from particle tracing visualized by the laser light sheet and compared to the predictions from equation (3). The measured velocities are typically smaller by a factor of 2 to 3 . Interestingly, this is similar to the measurements of migration velocities reported by Barton and Subramanian (1989). The flow Reynolds numbers for the present experiments were very low (i.e., $\mathrm{Re}<<1$ ). The Peclet numbers ranged 
from 1 to 9. Figure 6 shows a long time exposure photograph of the largest section of a vegetable oil droplet showing the pathlines of the thermocapillary flow traced by the seed particles.

While the droplets migrate farther away than predicted by linear theory (Figure 5), the interface velocities seem to be smaller (Table 2). Hence, stronger thermocapillary flow does not seem to be the reason for the excessive migration. By elimination, the larger migration distance must be because the effects of buoyancy are reduced compared to what linear theory anticipates. We speculate that this may be due to the following reasons: (i) The temperature distribution in and around the droplet (and consequently the density distribution and the buoyancy force) is different from linear theory predictions due to convection of energy. As mentioned before, the Peclet numbers range from 1 to 9 . Effects due to energy convection are not negligible and the isotherms would be distorted compared to those in pure conductive heat transfer. Since we have not measured the temperature distribution in the presence of the droplets, we do not know what the isotherms look like and cannot evaluate whether buoyancy effects have increased or decreased compared to what linear theory anticipates. Also, to our knowledge there is no theoretical analysis predicting the temperature distribution for conditions of the current experiments. (ii) as mentioned before, the droplets at equilibrium positions were located 3 to 10 radii from the bottom wall of the test cell, with the bigger droplets being closer to the wall than the smaller droplets. Consequently, wall interactions may not be negligible, especially for the larger droplets. The analysis by the Merritt and Subramanian (1989) shows that for an isolated bubble that is stationary under the action of buoyancy and thermocapillary forces, wall interaction effects 
tend to move the bubble in the direction of the temperature gradient. That is, the presence of walls appears as though the thermocapillary forces are enhanced (or the buoyancy forces are reduced), up to separation distances of even 10 to 15 radii. No theory seems to exist for the case of droplets. Our experiments seem to indicate that precisely such a phenomenon may be occurring; (iii) natural convection effects have no contributions to the force balance on the droplet in linear regimes as the temperature distribution in conductive heat transfer in the vicinity of the droplet is symmetric about its mid plane. However, when convection is important, as in the present experiment, natural convection in the host fluid is expected to play a role. This is not included in the linear theory. Once again, since we have not measured the temperature distribution near the droplet we are unable to evaluate whether the contribution from natural convection enhances or reduces the migration of the droplet; and (iv) lastly, the effects of mass transfer for short times are unknown and may have an influence; mass transfer definitely influences the droplet motion for long times.

In view of the lack of a theory which also considers the convective terms in the energy equation, an attempt has been made to describe a unified empirical fit to represent results of these experiments. Applying a multiple linear regression (Neville and Kennedy, 1964), using $\frac{1}{D}$ and $\frac{d T}{d z}$ as two independent variables, a fit has been calculated for the three different temperature gradients and droplet diameters, and they are shown in Figure 7. The empirical equation is expressed as follows 


$$
T_{\text {eq }}-T_{\mathrm{m}}=K\left[\left(\frac{1}{D}\right)^{\mathrm{a}}\right]\left[\left(\frac{d T}{d z}\right)^{\mathrm{b}}\right]
$$

where $\mathrm{a}=-0.7903$ and $\mathrm{b}=1.639$. The linear theory curves for droplets of 3 and $9 \mathrm{~mm}$ sizes are also plotted in the same coordinate system for a comparison. The value of the theory of Young . could be approached with decreasing temperature gradient and increasing the droplet size. Again, the trend is qualitatively the same, but different in magnitude.

While attempts have been made to calculate the thermocapillary migration of bubbles in zero gravity conditions (Szymczyk et al., 1987, Shankar and Subramanian, 1988, Balasubramaniam and Lavery, 1989), there do not appear to be any studies under normal gravity for droplets for non-zero Reynolds and Marangoni numbers. These calculations would have to be carried out to compare with the experimental results.

It is also well known that the motion of drops and bubbles is affected by surfactants which can adsorb on the interface and influence the interfacial tension coefficient (Harper, 1972). In experiments with water, Thompson et al. (1980) attribute the lack of motion of gas bubbles in a temperature gradient to the possible contamination of water by surface active impurities. The above consideration prompted a flow visualization experiment using droplets of water-methanol mixture (W-M, $50-50$ by weight) in the $5 \mathrm{CS}$. silicone host-liquid. Using laser flow visualization, the thermocapillary flow inside the droplets were compared for W-M and vegetable oil, for comparable temperature gradients and droplet sizes. Even though the vegetable oil is 
much more viscous than the water-methanol mixture, a flow pattern (Figure 6) resembling Hill's spherical vortex (Harper, 1972) was easily seen in vegetable oil droplets. In contrast, both the surface flow and the return flow in W-M seemed to be considerably retarded.

\section{Conclusions}

Thermocapillary flow inside and on the interface of immiscible droplets in a host fluid have been, for the first time, visualized with directions consistent with the sign of the measured thermal interfacial tension coefficient (i.e., $\frac{d \sigma}{d T}<0$ ). The flow persisted for long times. For short times after injection, droplets migrate toward the hot upper wall. Mass transfer affects the migration of the droplets for longer times. Our experimental results follow the linear theory predictions qualitatively, but are quantitatively different. The droplets migrated farther away from the density-matched temperature compared to linear theory predictions. This appears to be not because of stronger thermocapillary flows, but due to reduced effects of buoyancy. We speculate that this may be due to (i) effects of convective heat transfer as the Marangoni numbers are non-negligible, (ii) wall interaction effects, (iii) natural convection effects which have not been accounted for in the linear theory, and finally (iv) the effects of mass transfer for short times that are unknown. Flow visualization studies in droplets of water-methanol mixture in silicone oil have revealed a significantly retarded flow pattern, compared to vegetable oil droplets. This suggests possible surfactant influence in the water-methanol mixture. 
Based on our experience, reduced gravity experiments are perhaps the only way to provide direct and unambiguous migration data for non-negligible Marangoni numbers.

\section{References}

Barton, K. D. \& Subramanian, R. S. (1989) "The Migration of Liquid Drops in a Vertical Temperature Gradient," to be published in J. of Colloid and Interface Science.

Balasubramaniam, R. \& Lavery, J. (1989) "Numerical Simulation of Thermocapillary Bubble Migration Under Microgravity for Large Reynolds and Marangoni Numbers," Numerical Heat Transfer, Part A, 16, 175 .

Chifu, E., Stan, I., Finta, Z. \& Gavrila, E. (1983) "Marangoni-Type Surface Flow on an Undeformable Free Drop," J. Coll. Interface Sci. 93, 140.

Delitzsch, V., Eckelmann, H. \& Wuest, W. (1984) "Migration of Droplets Under Reduced Gravity," Proceedings of the 5th European Symposium on Material Sciences under Microgravity, Schloss Elmanu.

Hardy, S. C. (1979) "The Motion of Bubbles in a Vertical Temperature Gradient," J. Colloid Interface Sci. 69, 157.

Harper, J.F. (1972) "The Motion of Bubbles and Drops Through Liquids," Advances in Applied Mechanics, 12, 59, Academic Press, London.

Merritt, R. M., \& Subramanian, R. S. (1989) "Migration of a Gas Bubble Normal to a Plane Horizontal Surface in a Vertical Temperature Gradient," J. Colloid Interface Sci, 131,514.

Năhle, R., Neuhaus, D., Siekmann, J., Wozniak, G., \& Srulijes, J. (1987) "Separation of Fluid Phases and Bubble Dynamics in a Temperature Gradient - a Spacelab D1 Experiment," Z. Flugwiss. Weltraumforsch $11,211$.

Neilson, G. F. \& Weinberg, M. C. (1977) "Outer Space Formation of a Laser Host Glass," Journal of Non-Crystalline Solids 23, 43.

Neville, A. M. \& Kennedy, J. B. (1964) "Basic Statistical Methods for Engineers and Scientists," International Textbook Company.

Papazian, J. M. \& Wilcox, W. R. (1978) "Interaction of Bubbles with Solidification Interfaces," AIAA J., 16, 447-451.

Perry, R.H. \& Chilton, C.H. (1973) "Chemical Engineering Handbook," 5th edn. McGraw-Hill Company. 
Rashidnia, N. \& Balasubramaniam, R. (1989) "Interfacial Tension Measurement of Matched-Density Liquids," in progress.

Seikmann, J., Wozniak, G., Srulijes, J., Nähle, R. \& Neuhaus (1986) "Experimental Investigation of Thermocapillary Bubble and Drop Motion Under Microgravity," Proc. 6th European Symposium on Material under microgravity conditions, Bordeaux, France.

Shankar, N. \& Subramanian, R. S. (1988) "The Stokes Motion of a Gas Bubble Due to Interfacial Tension Gradients at Low to Moderate Marangoni Numbers," J. Colloid Interface Sci. 123, 512.

Szymczyk, J. A., Wozniak, G. \& Siekmann, J. (1987) "On Marangoni Bubble Motion at Higher Reynoldsand Marangoni-Numbers Under Microgravity." Appl. Microgravity Tech. 1, 1.

Subramanian, R. S. (1985) "The Stokes Force on a Droplet in an Unbounded Fluid Medium Due to Capillary Effects," J. Fluid Mech. 153, 389.

Thompson, R. L. (1979) "Marangoni Bubble Motion in Zero Gravity," Ph. D. Thesis, Chemical Engineering, University of Toledo.

Thompson, R. L., Dewitt, K. J., \& Labus, T. L. (1980) "Marangoni Bubble Motion Phenomenon in Zero Gravity," Chem. Eng. Commun. 5, 299.

Weinberg, M. C., March 22 (1978) Glass Ind.

Young, N. O., Goldstein, J. S. \& Block, M. J. (1959) "The Motion of Bubbles in a Vertical Temperature Gradient," J. Fluid Mech. 6, 350. 
Table 1

Properties of Liquids

\begin{tabular}{|lllllll|}
\hline Froperty & $\begin{array}{c}\rho \mathrm{g} / \mathrm{cc} \\
\text { at } \\
T_{\mathrm{m}}=15^{\circ} \mathrm{C}\end{array}$ & $\beta \frac{1}{{ }^{\circ} \mathrm{C}}$ & $v c s$. & $k \frac{\mathrm{cal}}{\mathrm{cm} \mathrm{sec}{ }^{\circ} \mathrm{C}}$ & $a \frac{\mathrm{cm}^{2}}{\mathrm{sec}}$ & $\operatorname{Pr}$ \\
\hline $\begin{array}{l}\text { Sillicon } \\
\text { Oil }\end{array}$ & 0.911 & 0.0001197 & 5 & 0.00026 & 0.0007 & 71.75 \\
\hline $\begin{array}{l}\text { Vegetable } \\
\text { Oil }\end{array}$ & 0.911 & 0.000703 & 66.9 & 0.0003766 & 0.00077 & 868.83 \\
\hline
\end{tabular}

Table 2

Measured Data and Comparison with the Linear Theory Predictions

\begin{tabular}{|c|c|c|c|c|c|c|}
\hline $\begin{array}{l}\frac{d T}{d z} \\
\frac{{ }^{\circ} C}{c m}\end{array}$ & $\begin{array}{l}\mathrm{D} \\
m m\end{array}$ & $\begin{array}{c}V_{\mathbf{m}} \\
\text { MEASURED } \\
\mu_{\boldsymbol{m}} / \mathrm{sec}\end{array}$ & $\begin{array}{c}V_{\mathrm{p}} \\
\text { PREDICTED } \\
\text { LT. } \\
\mu_{m} / s e c\end{array}$ & $\frac{v_{p}}{v_{m}}$ & $\frac{V_{m} D}{2 \alpha}$ & $\frac{\rho \frac{d \sigma}{d T} \frac{d T}{d z} D^{2}}{4 \mu^{2}} \operatorname{Pr}$ \\
\hline 23.63 & 8.0 & 143.8 & 390.4 & 2.72 & 8.25 & 1049.3 \\
\hline 19.81 & 7.6 & 105.4 & 311.0 & 2.95 & 5.75 & 793.9 \\
\hline 14.35 & 7.63 & 84.0 & 226.2 & 2.69 & 4.60 & 579.9 \\
\hline 23.63 & 8.0 & 143.8 & 390.4 & 2.72 & 8.25 & 1049.3 \\
\hline 23.63 & 5.45 & 125.6 & 266.0 & 2.12 & 4.90 & 487.0 \\
\hline 23.63 & 4.1 & 93.1 & 200.1 & 2.15 & 2.74 & 275.6 \\
\hline
\end{tabular}




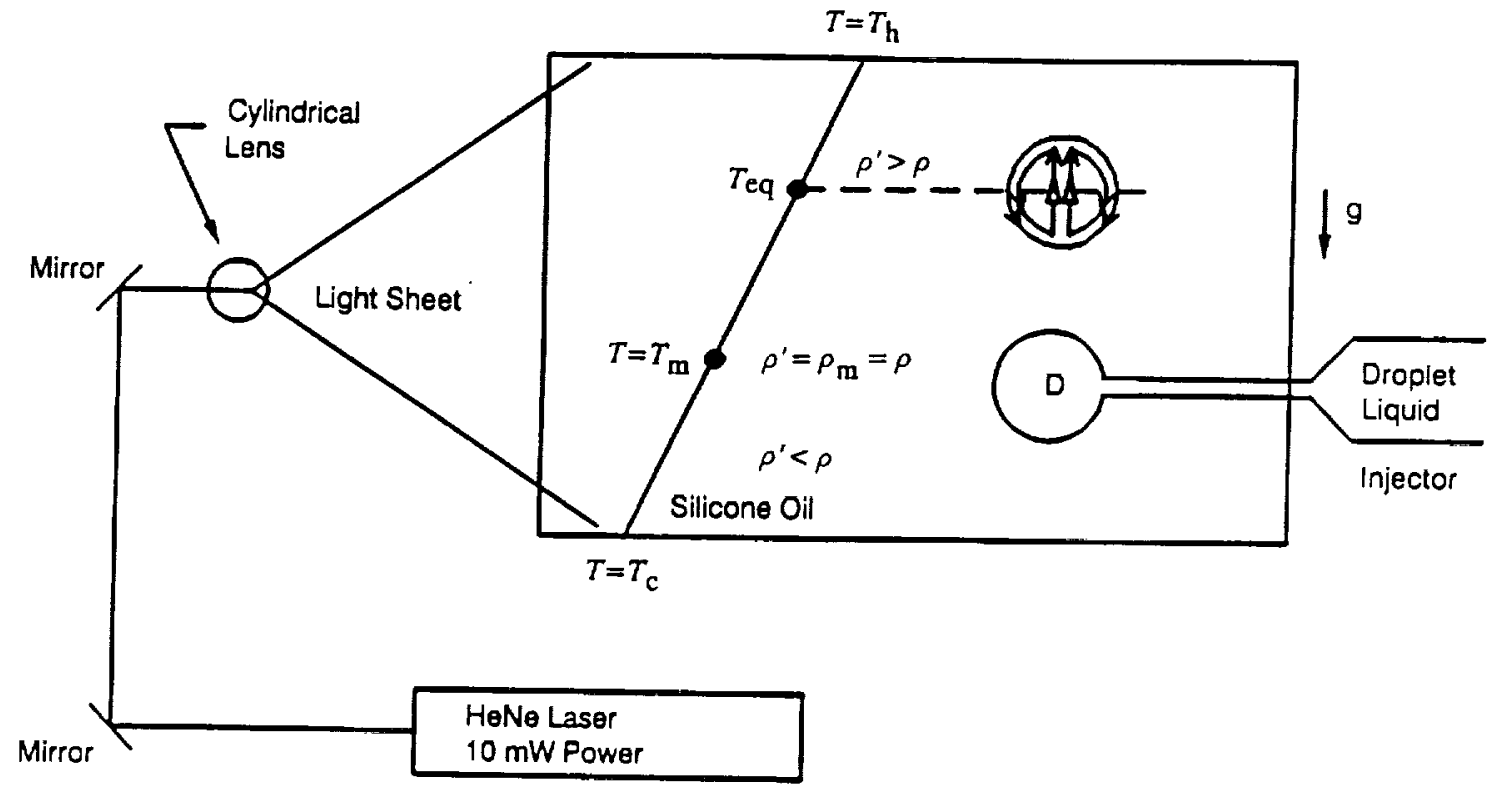

Figure 1

Schematic of the Matched-Density Experiment

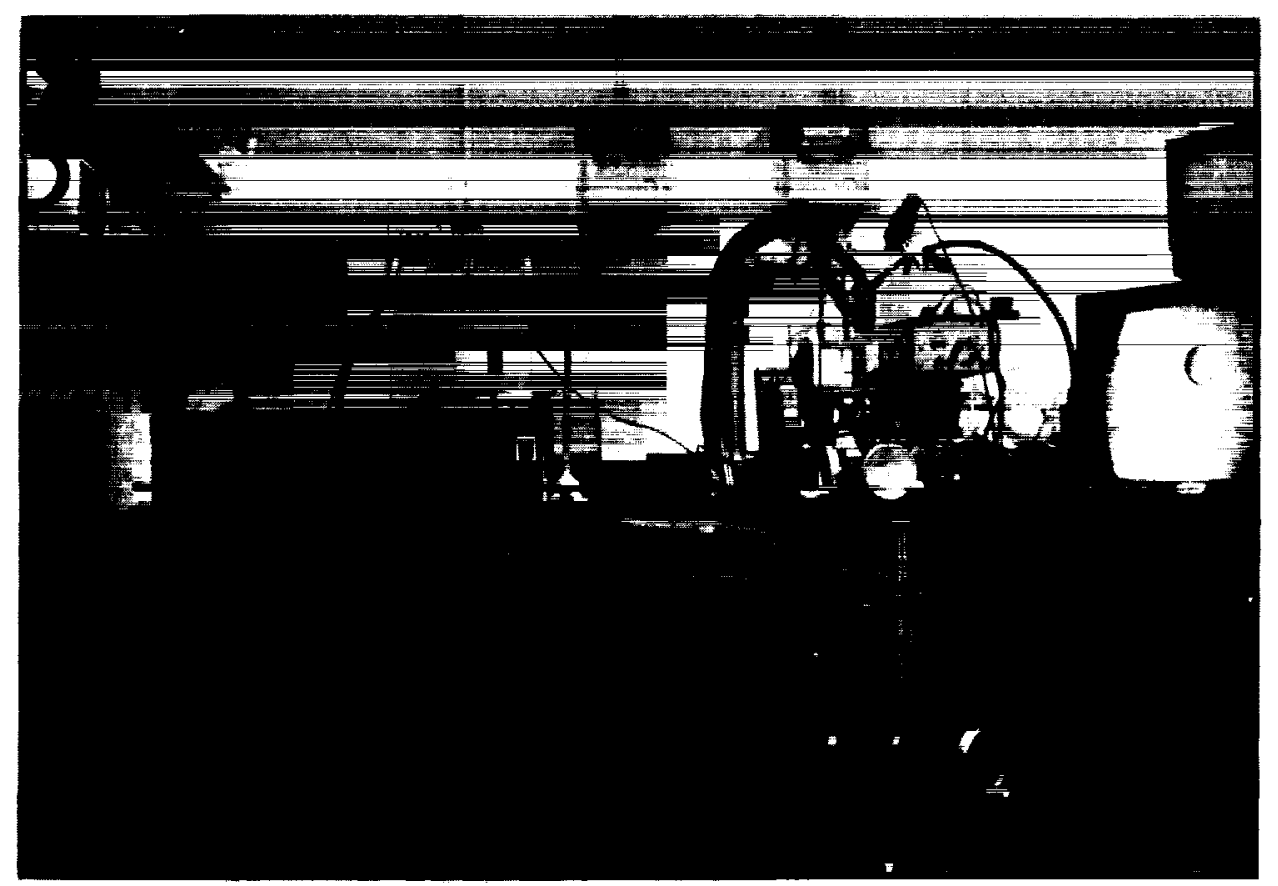

Figure 2

Experimental Apparatus 


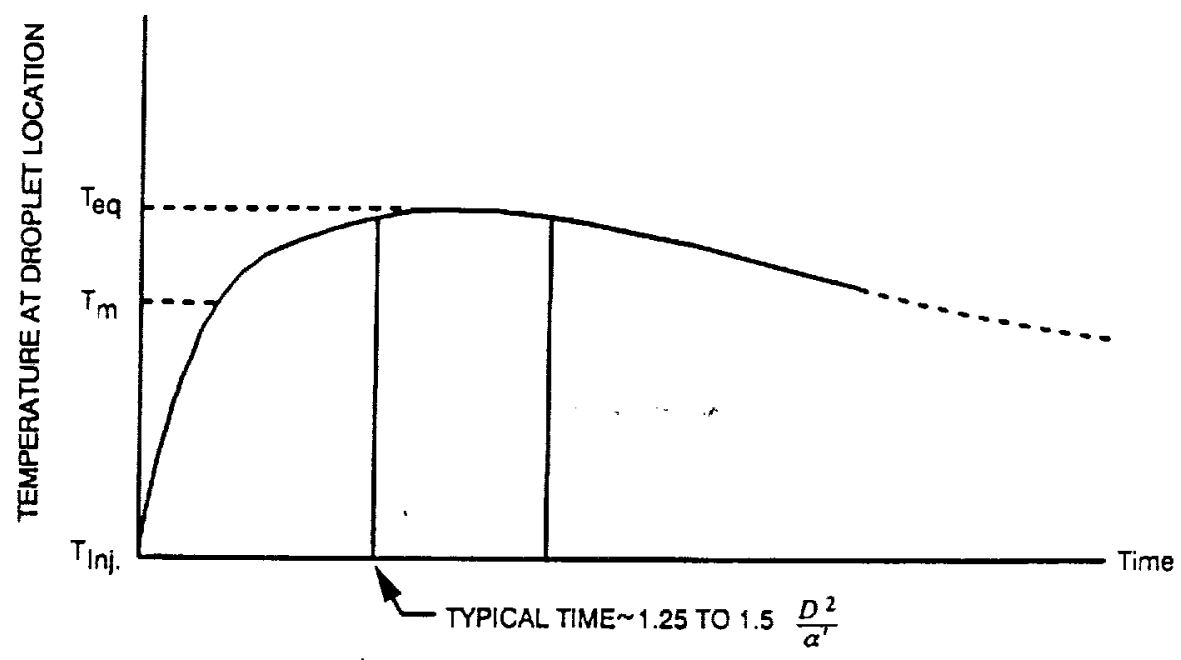

Figure 3

Typical Droplet Temperature vs Time

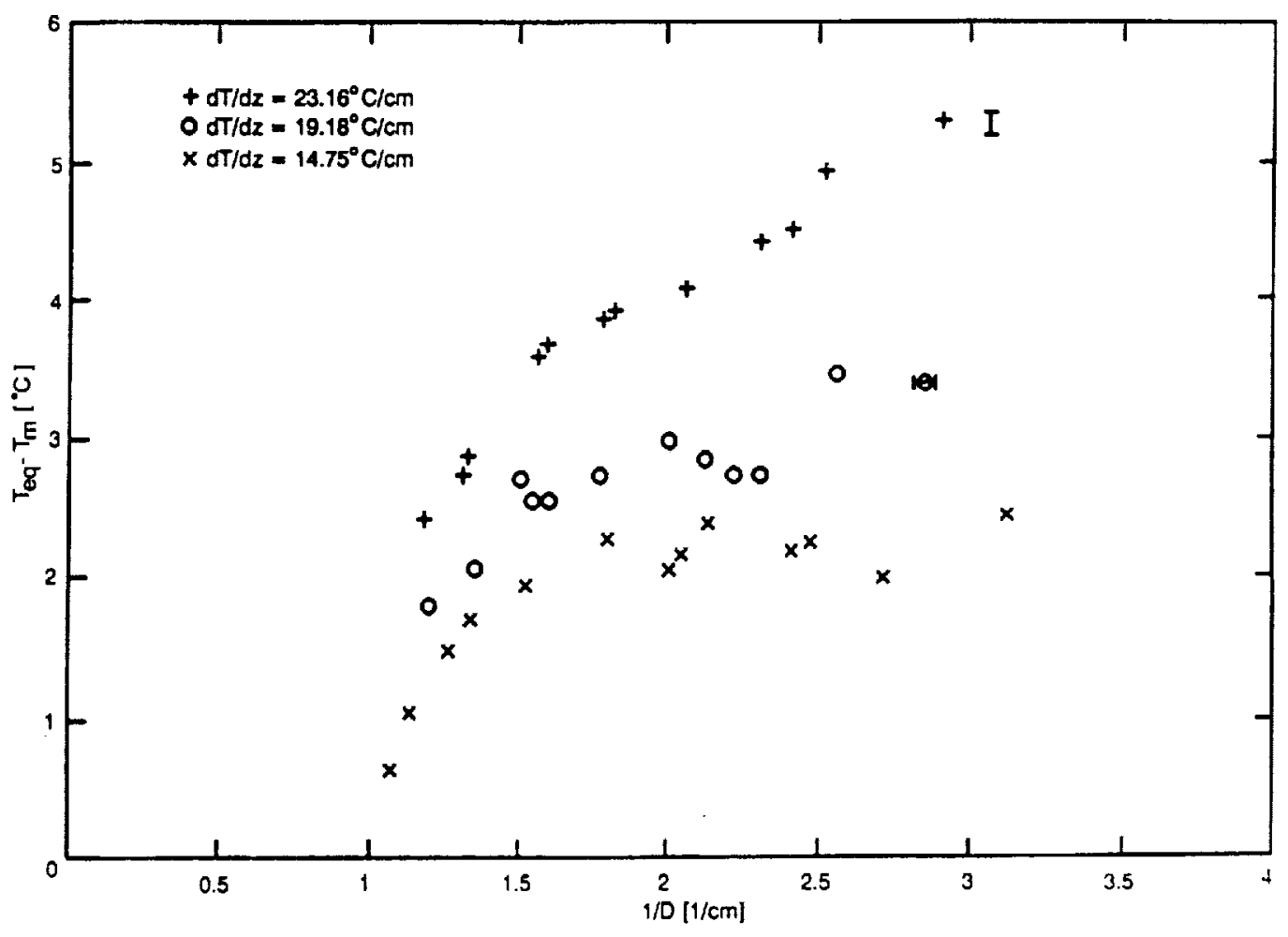

Figure 4

Raw Data 


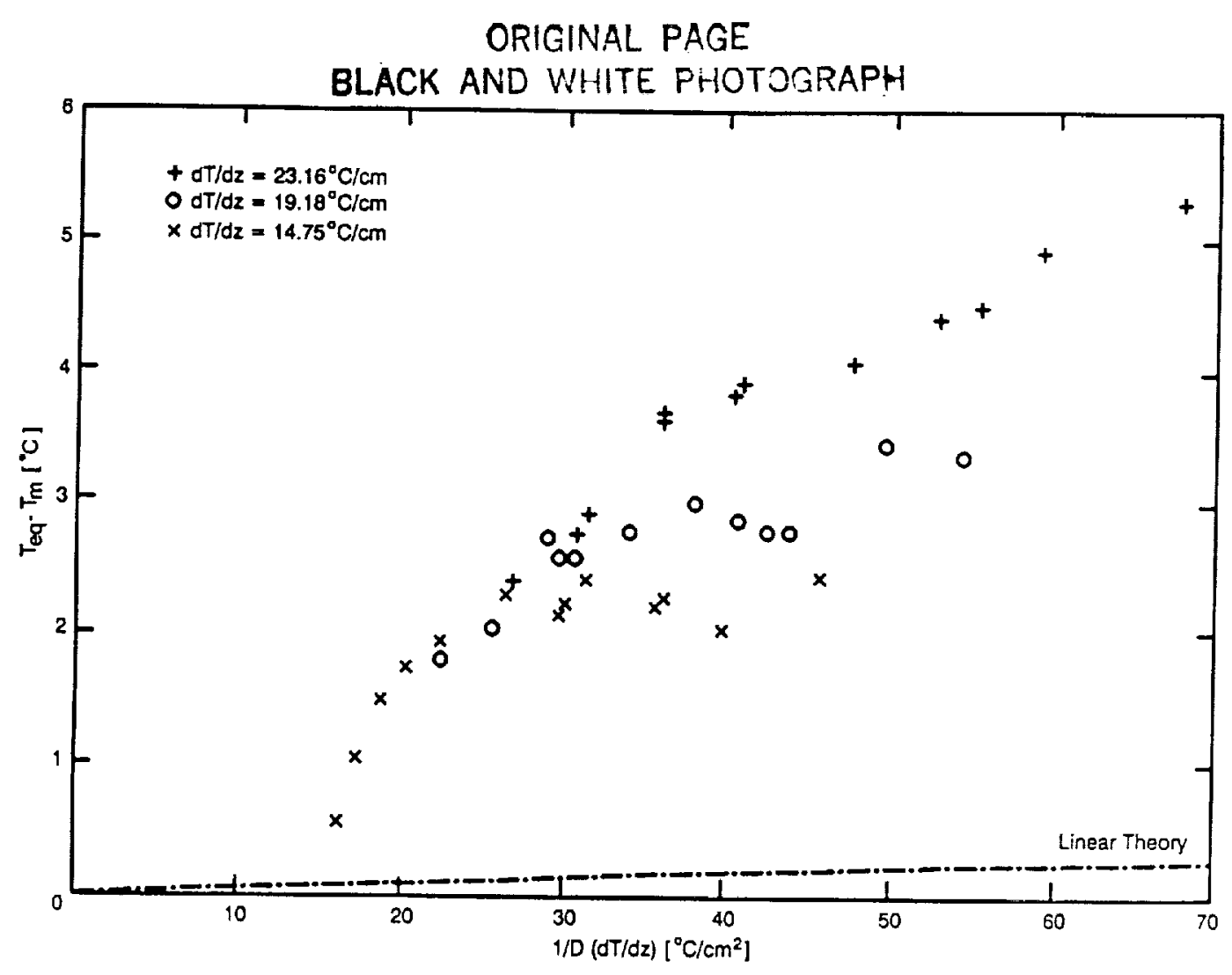

Figure 5

Comparison of Present Results with Linear Theory Predictions

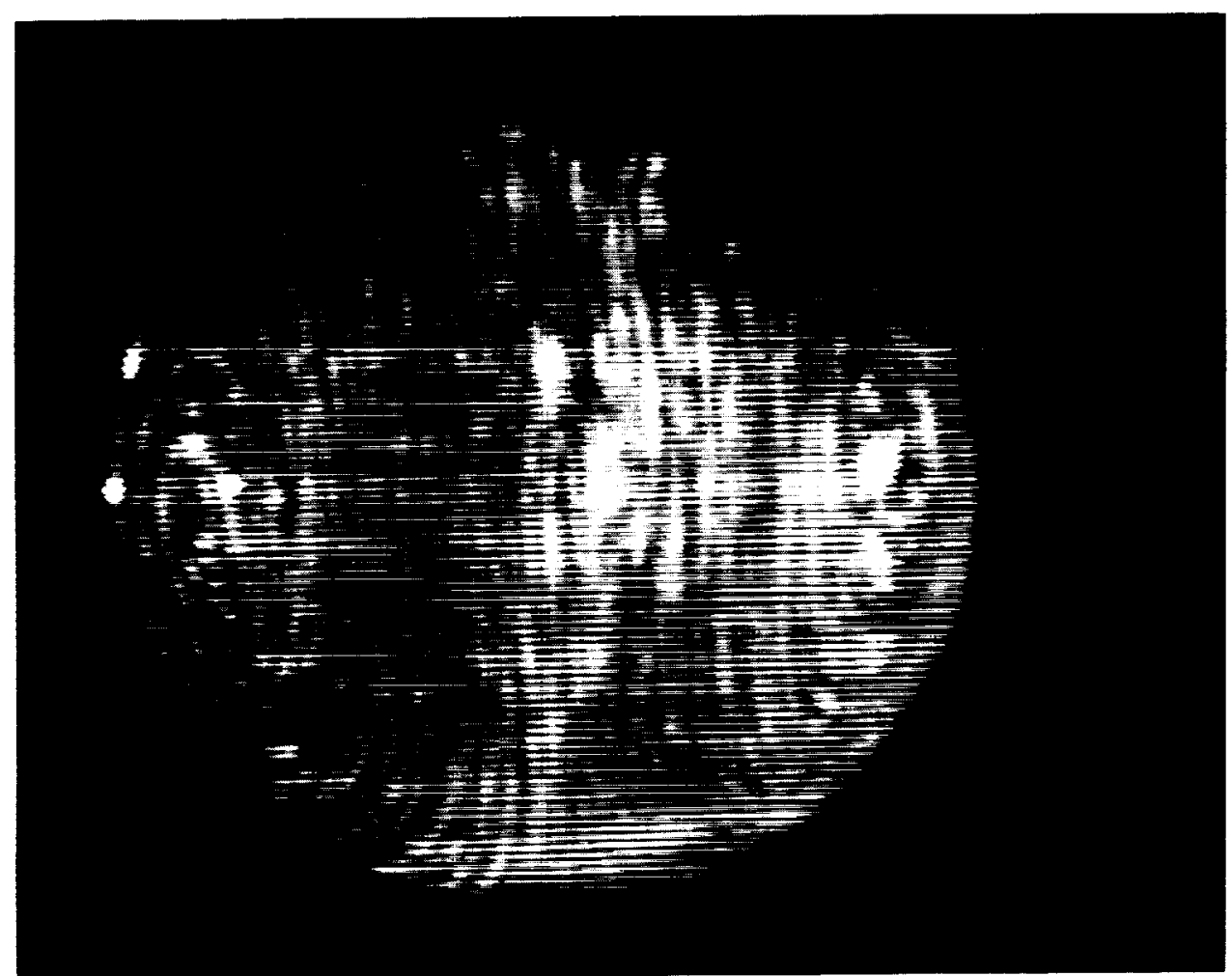

Figure 6

Long-Time Exposure Photo of Flow Inside a Droplet $\left(D=9 \mathrm{~mm}, \mathrm{dT} / \mathrm{dz}=6^{\circ} \mathrm{C} / \mathrm{cm}\right)$ 


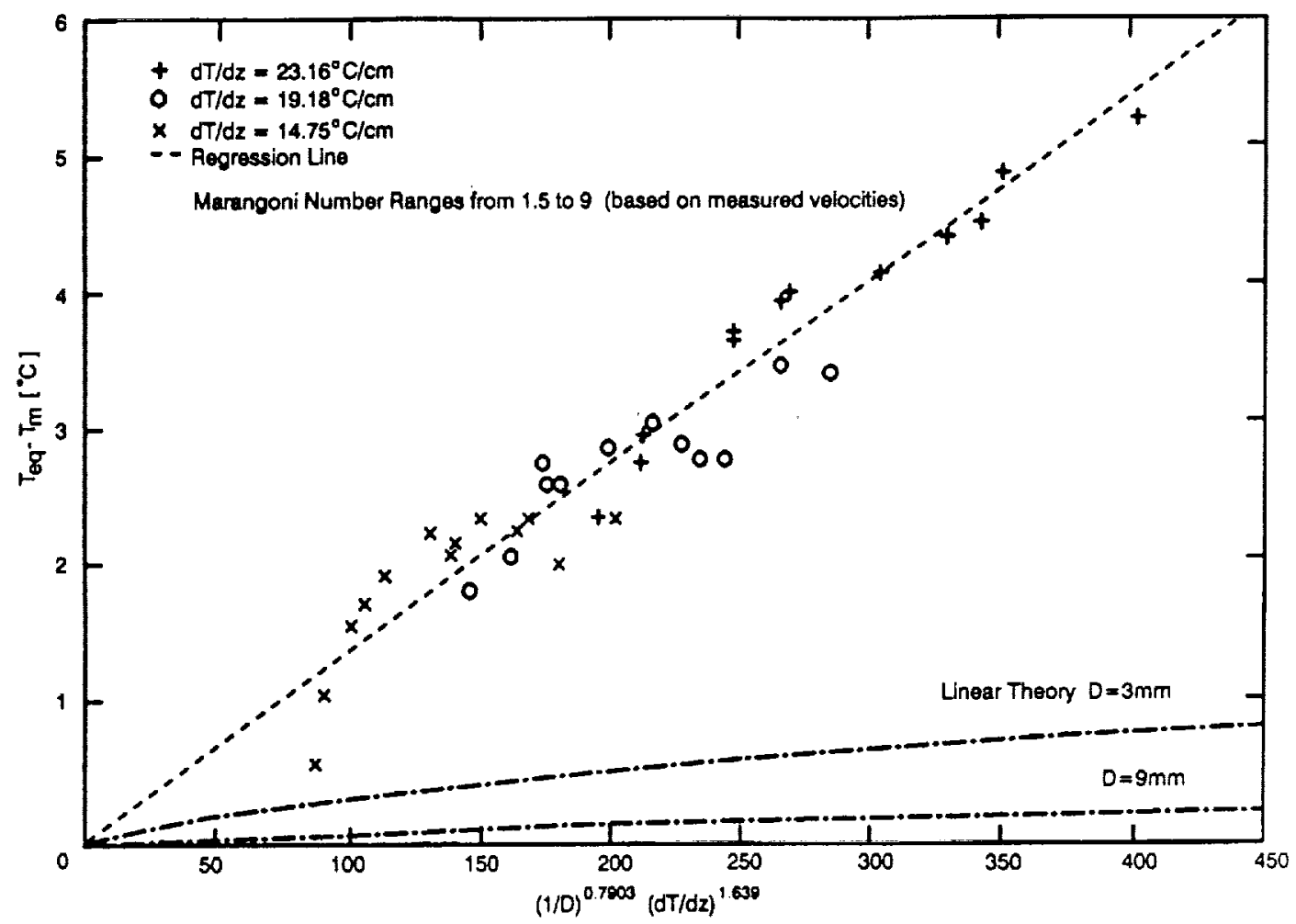

Figure 7

Unified Curve-Fitted Results and Comparison with Linear Theory 


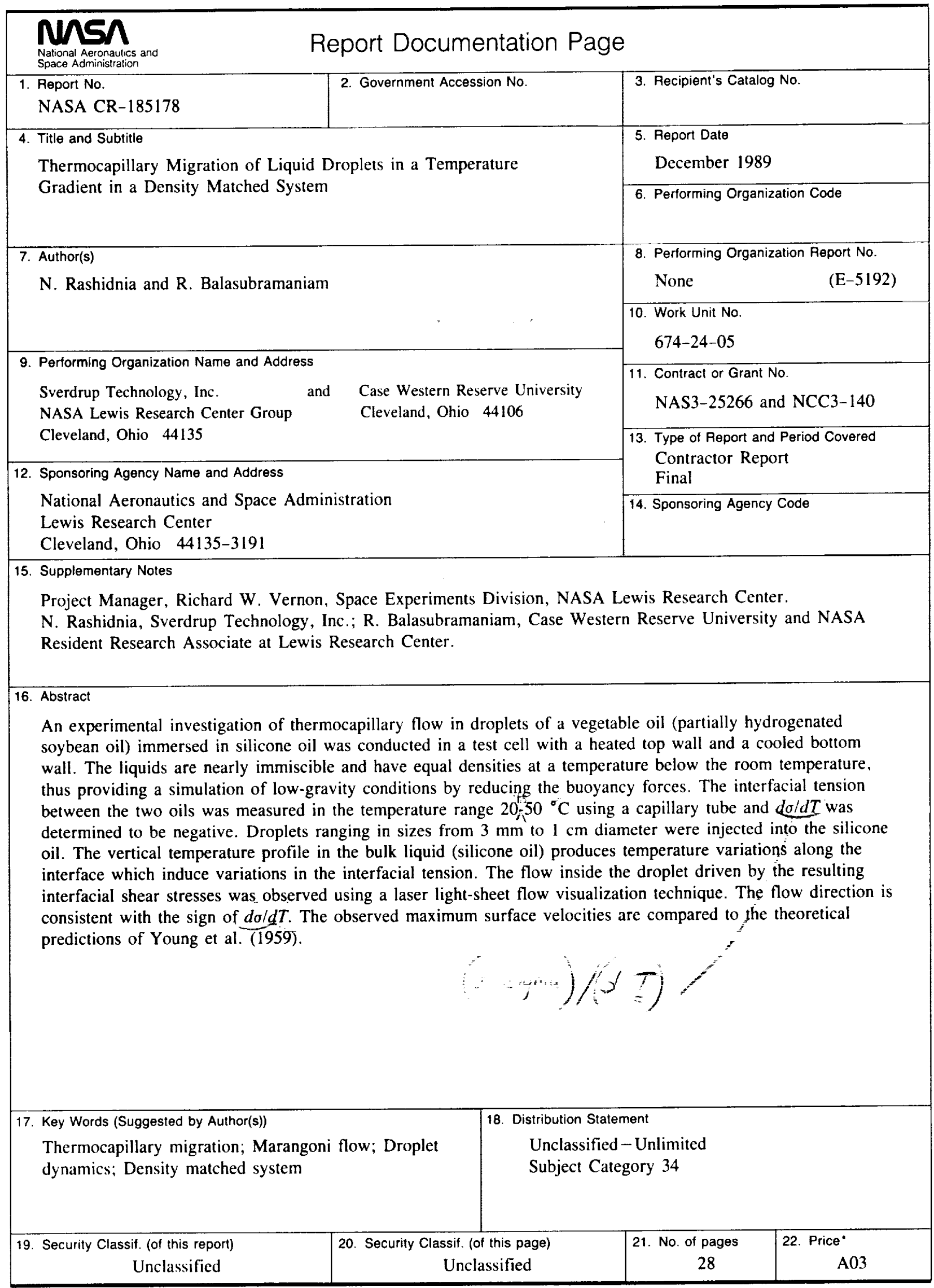


